\title{
Editorial Overview: Surface electrochemistry
}

\section{Marc T.M. Koper}

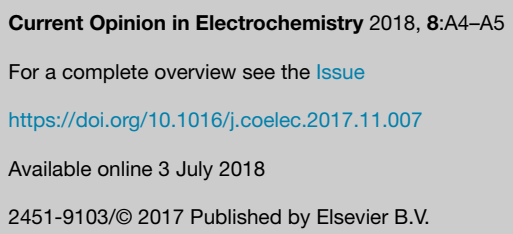

\section{Marc T.M. Koper}

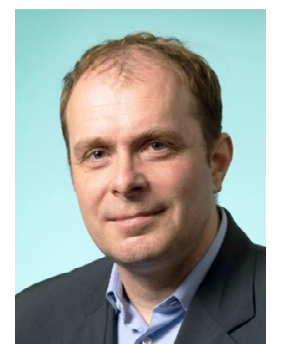

Marc T.M. Koper is Professor of Fundamental Surface Science at the Leiden Institute of Chemistry of Leiden University (The Netherlands). His research interests are in fundamental aspects of surface electrochemistry, electrocatalysis and theoretical and computational electrochemistry.
Electrochemical reactions and phenomena take place at surfaces and interfaces. Probing and understanding electrochemical events at interfaces are therefore at the core of surface electrochemistry, and of utmost importance for the description and design of electrochemical devices. This thematic issue of Current Opinion in Electrochemistry collects short reviews by leading experts in a number of established as well as emerging fields in surface electrochemistry. Theoretical and computational aspects of surface electrochemistry are covered in the contributions by Jinnouchi et al. [1] on electronic structure calculations of electrode/electrolyte interfaces, and by Calle-Vallejo et al. [2] on adsorption energy scaling relations in electrocatalytic reaction mechanisms. State-of-the-art developments in spectroscopy and imaging of electrochemical interfaces are reviewed by Domke et al. [3] (on Electrochemical Tip-Enhanced Raman Spectroscopy EC-TERS) and by Rodríguez-López et al. [4] (on Electrochemical Scanning Probe techniques), while Motobayahsi and Osawa [5] discuss recent advances in the spectroscopic characterization of electrochemical interfaces involving ionic liquid electrolytes. Advances in the surface electrochemical study of key electrocatalytic reactions are dealt with in the papers by Escudero-Escribano et al. [6] (oxygen reduction reaction) and Yeo et al. [7] (carbon dioxide reduction), while Cherevko [8] discusses important work on the surface mechanisms involved in the stability of catalysts for the oxygen reduction and oxygen evolution reactions. Surface electrochemistry also provides the essential tools for the examination of the electrochemical properties of surface-confined enzymes, as discussed by Jenner and Butt [9], and for supramolecular electrochemistry, as discussed by Mertens et al. [10]

I would like to thank all contributors for their great efforts in writing such excellent papers, and I am certain that the short reviews in this issue will be useful for all those readers who want to be updated in the latest state-ofthe-art developments in the topics covered. 


\section{References}

1. Jinnouchi R, Kodama K, Morimoto $Y$ : Electronic structure calculations on electrolyte-electrode interfaces: successes and limitations. Curr Opin Electrochem 2018, 8:103-109.

2. Govindarajan N, García-Lastra JM, Meijer EJ, Calle-Vallejo F: Does the breaking of adsorption-energy scaling relations guarantee enhanced electrocatalysis? Curr Opin Electrochem 2018, 8:110-117.

3. Pfisterer JHK, Domke KF: Unfolding the versatile potential of EC-TERS for electrocatalysis. Curr Opin Electrochem 2018, 8:96-102.

4. Schorr NB, Gossage ZT, Rodríguez-López J: Prospects for single-site interrogation using in situ multimodal electrochemical scanning probe techniques. Curr Opin Electrochem 2018, 8:89-95.

5. Motobayashi K, Osawa M: Recent advances in spectroscopic investigations on ionic liquid/electrode interfaces. Curr Opin Electrochem 2018, 8:147-155.
6. Escudero-Escribano M, Jensen KD, Jensen AW: Recent advances in bimetallic electrocatalysts for oxygen reduction: design principles, structure-function relations and active phase elucidation. Curr Opin Electrochem 2018, 8:135-146.

7. $\quad$ Ting LRL, Yeo BS: Recent advances in understanding mechanisms for the electrochemical reduction of carbon dioxide. Curr Opin Electrochem 2018, 8:126-134.

8. Cherevko S: Stability and dissolution of electrocatalysts: Building the bridge between model and "real world" systems. Curr Opin Electrochem 2018, 8:118-125.

9. Jenner LP, Butt JN: Electrochemistry of surface-confined enzymes: inspiration, insight and opportunity for sustainable biotechnology. Curr Opin Electrochem 2018, 8:81-88.

10. Cui K, Dorner I, Mertens SFL: Interfacial supramolecular electrochemistry. Curr Opin Electrochem 2018, 8:156-163. 